MODELING, IDENTIFICATION AND CONTROL, 1995, VOL. 16, NO. 1, 51-63

doi:10.4173/mic.1995.13

\title{
Elementary nonlinear decoupling control of composition in binary distillation columns
}

\author{
JENS G. BALCHEN $\dagger$ and BJARNE SANDRIB $\dagger$ \\ Keywords: Nonlinear control, decoupling, distillation, composition. \\ Elemenatary nonlinear decoupling (END) is a model based control algorithm which \\ intends to decouple and linearize a nonlinear multivariable process in order to \\ achieve better control than can be obtained by conventional decentralized linear \\ feedback control. The application of END to the composition control of a theoretical \\ binary distillation column illustrates that the achievable quality is very high.
}

\section{Introduction}

The control of composition in binary distillation columns has been the subject of research for many years and a large number of contributions with different control strategies have been presented. A comprehensive review of such contributions is given in Skogestad (1993).

One control strategy which has so far not been tested for the composition control of binary distillation columns is nonlinear decoupling. This control strategy attempts to linearize and decouple (diagonalize) the process in order that high performance linear control can be applied (Isidori 1989, Balchen et al. 1987, Balchen 1991, Balchen 1993).

Since a distillation column controlled by the reflux of liquid top product and boil-up vapour flow of bottom product is highly nonlinear and has a large number of state variables the theory of elementary nonlinear decoupling (END) introduced by Balchen (1993) seems very appropriate.

\section{Elementary nonlinear decoupling (END)}

END is a version of the original nonlinear decoupling algorithm that solves the invertibility problem by designing the property space which is the object of the linearization and decoupling. It has some similarity with the input-output linearization (Isidori 1989) in which the invertibility problem is solved by differentiating the output variables a number of times. In Balchen (1993) it is claimed that the END is the most realistic solution in practical systems.

A nonlinear dynamic process is described by

$$
\dot{\boldsymbol{x}}=f(x, u, v)
$$

where $x, u, v$ are state-, control- and disturbance vectors respectively and $f$ is a set of nonlinear functions.

Received 30 August 1994.

$\dagger$ The Norwegian Instiute of Technology, Department of Engineering Cybernetics, 7034 Trondheim, Norway.

Reprinted from the preprints of the IFAC Symposium on Advanced Control of Chemical Processes (ADCHEM), Kyoto, Japan with kind permission from Pergamon Press Ltd. 
In a distillation column the dimension $(n)$ of the state vector will usually be very much larger than the dimension $(r)$ of the control vector. This has severe conseguences in the development of most model based control strategies. As will be shown in the following, the consequences in END are less pronounced.

A common phenomenon in nonlinear modeling of dynamic processes is that the system is only nonlinear in the states and disturbances but linear in the control variable such that

$$
\dot{x}=f(x, v)+B(x) u
$$

In END one defines a property transformation $z=d(x)$ which is commonly replaced by a simple linear transformation

$$
z=D x
$$

in which the property vector $z$ may be interpreted as the properties one wants to control.

END intends to determine the control action $(u)$ which will drive the dynamic system in such a way that $\dot{z}$ follows a desired trajectory $\dot{z}_{d}$.

From (2) and (3) it follows that

$$
\dot{z}=D \dot{x}=D f(x, v)+D B(x) u
$$

(4) can be solved with respect to $u$ yielding

$$
u=(D B(\cdot))^{-1}\left(\dot{z}_{d}-D f(x, v)\right)
$$

in which $\dot{z}$ has been replaced by $\dot{z}_{d}$.

Equation (5) will only have a solution as long as the matrix $D B(\cdot)$ has an inverse. That puts restrictions on both $D$ and $B$. The first restriction is that $D B(\cdot)$ must be a square matrix implying that $\operatorname{dim} z=\operatorname{dim} u$. If $\operatorname{dim} u>\operatorname{dim} z$ one has an optimization problem which will not be dealt with here.

Since in general $B(\cdot)$ is given for a certain process, one has to design the $\mathbf{D}$ matrix such that $D B(\cdot)$ becomes non-singular and the resulting system has acceptable dynamic properties.

Applying (5) and (2) yields

$$
\dot{x}=\left(I-B(D B)^{-1} D\right) f(x, v)+B(D B)^{-1} z_{d}
$$

which when linearized becomes

$$
\dot{\boldsymbol{x}}=\left(I-B(D B)^{-1} D\right)(A x+C v)+B(D B)^{-1} z_{d}
$$

assuming for simplicity that $B(x)=B=$ constant. Multiplying (6) by $D$ gives

$$
\dot{\boldsymbol{z}}=\boldsymbol{D} \dot{\boldsymbol{x}}=\dot{\boldsymbol{z}}_{d}
$$

which shows that the system has been decoupled and linearized and is replaced by $r$ integrators between $\dot{z}_{d}$ and $z$.

$\dot{z}_{d}$ can be regarded as the new control input and $z$ as the new property output.

The dynamics of the system is determined by the differential equation of (6) and approximately by (7) with eigenvalues of the matrix

$$
\left(I-B(D B)^{-1} D\right) A
$$

characterizing the system stability.

From (7) the main system transfer matrix is given by

$$
x(s)=H(s) \dot{z}_{d}(s)
$$




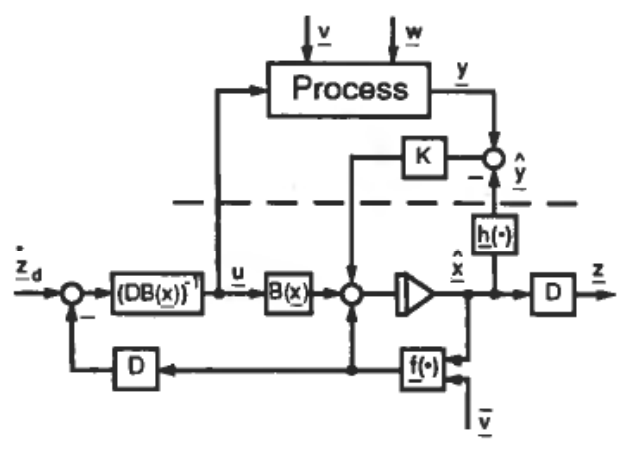

Figure 1. Nonlinear decoupling structure.

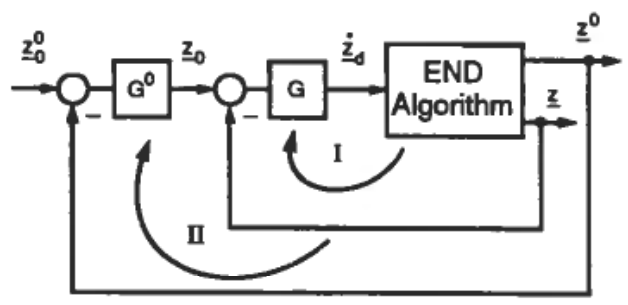

Figure 2. Feedback control loops.

where

$$
H(s)=\left(s I-\left(I-B(D B)^{-1} D\right) A\right)^{-1} B(D B)^{-1}
$$

This transfer matrix converted into the frequency response matrix $H(j \omega)$ can be characterized by means of its maximal and minimal singular value $\bar{\sigma}_{H}(j \omega)$ and $\sigma_{H}(j \omega)$. This gives a method for designing dynamic behaviour by choosing different $D$ matrices.

The contents of (5) and (3) can be expressed in a block diagram as shown in Fig. 1. The blocks below the dashed line reflect the mathematical model of the process and as can be seen, the full state $(\boldsymbol{x})$ and the disturbance $(\boldsymbol{v})$ are assumed to be available. In order to achieve that, a state estimator is employed which may be e.g. an Augmented Kalman filter based on the measurements $(y)$. Note that the quantity $\bar{v}$ acting upon the nonlinear function, represents the measurable part of the process disturbance (v). In this way feed forward is realized in a very direct and correct manner.

The design of the property transformation $D$ has the goal of making the system invertible with acceptable dynamic properties. One could think of another transformation $z^{0}=D^{0} x$ which expresses the desirable but nonrealizable output vector. The reason for the nonrealizability is that the matrix $D^{0} B$ most often is singular.

When introducing the difference $\Delta z=z-z^{0}$ and the frequency response matrix $\Delta H(j \omega)$ defined by

$$
\Delta z(j \omega)=\Delta H(j \omega) \dot{z}_{d}(j \omega)
$$

one can find the transformation matrix $\mathbf{D}$ which minimizes some scalar measure of the matrix $\Delta H(j \omega)$. Such a measure is the $H_{\infty}$-norm given by $-\max _{\omega} \bar{\sigma}_{\Delta H}(j \omega)$. In the following the design of $\mathrm{D}$ is done in a rather simple manner by choosing a matrix which only has a few elements different than those of $D^{0}$ such that the dynamic behaviour of the system is acceptable. 

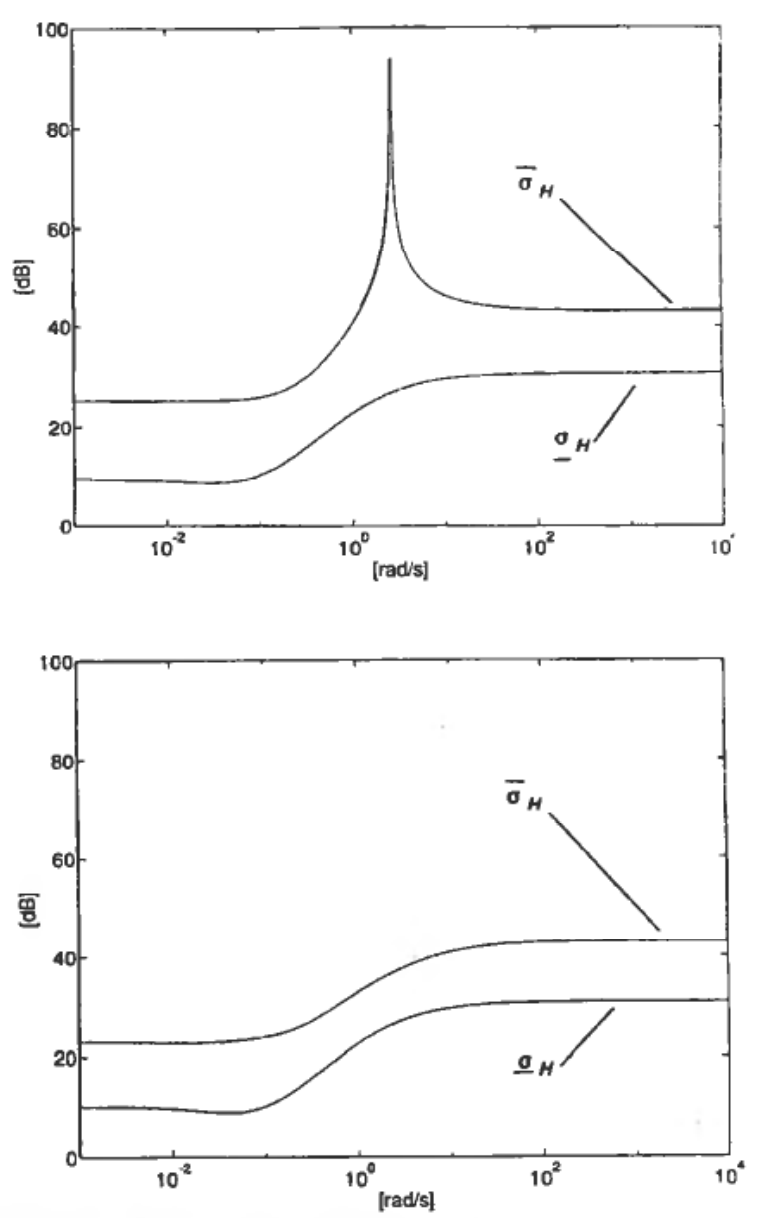

Figure 3. Maximal and minimal singular values from $\dot{z}_{d}$ to $\dot{\boldsymbol{x}}$. (Lower curve implemented.)

Figure 2 shows the proposed feedback control around the system where the END algorithm has been applied. There is an inner loop (I) containing a control matrix $G$ and with a reference vector $z_{0}$ and output $z$. This loop will have a nearly perfect response with very high bandwidth. The outer feedback loop (II) containing a control matrix $G^{0}$, a reference vector $z_{0}^{0}$ and an output $z^{0}$ will be slightly degraded relative to loop (I) particularly with respect to bandwidth, but with a performance which is in many cases greatly improved compared to a system containing only loop (II).

\section{A dynamic model of a binary distillation column}

A number of state space models for distillation columns are available in the literature with varying degrees of details in the models. Skogestad (1993) presents arguments for different kinds of simplifications which may be done without losing too much of the important dynamics of the column. A common simplification is to neglect the vapour hold up and the energy hold up in the column. The important dynamic effects left in the model are liquid dynamics and composition dynamics. The result is a dynamic model with a reasonable complexity with $2 N+4$ state variables where $N$ is the number of trays in the column. This type of model presented in the Appendix will be assumed in the next paragraphs even though other models could just as well have been used. 

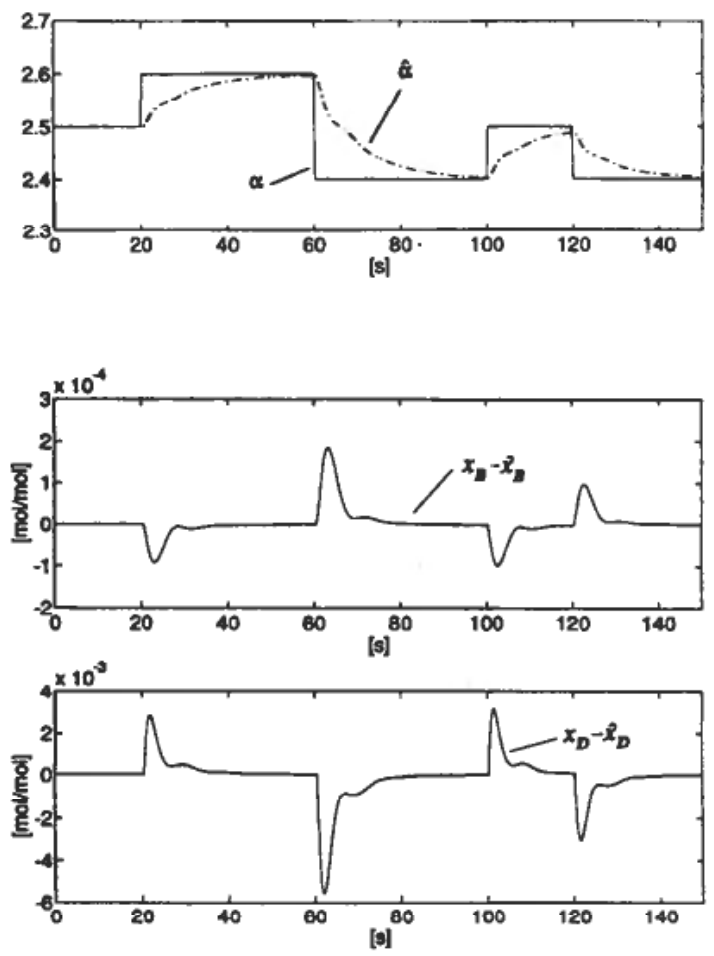

Figure 4. Estimator responses.

\section{Illustration of end control of a distillation column}

A simulation study has been performed using a distillation column as described in the appendix. The total system employing an Augmented Kalman Filter as shown in Fig. 1 and with feedbacks as shown in Fig. 2 is studied.

Figure 3 shows $\bar{\sigma}_{H}(j \omega)$ and $\sigma_{H}(j \omega)$ for two choices of $D$ as given in the appendix of which the latter is implemented in the system.

The performance of the Kalman filter is illustrated first without any nonlinear decoupling and feedback.

Figure 4 shows estimator responses to step changes in relative volatility $(\alpha)$ in the process.

In Fig. 5 nonlinear decoupling is employed and responses in $z, z^{0}$ and $u$ are shown following step changes in $\dot{z}_{d}$ (Fig. $5(a)$ ) and $v$ (Fig. $5(b)$ ).

In Fig. 6 diagonal, proportional control is employed in loop I. $z$ and the responses of the 'realizable' non-linear decoupling is illustrated following steps in $z_{0}$.

In Fig. 7 diagonal PI-control is employed in loop II and responses following step changes in $z_{0}^{0}$ (Fig. $7(a)$ ) and disturbances $v$ (Fig. 7(b)) are shown.

Figure 8 shows reponses of an ordinary well-tuned PI-two point controller (top concentration-reflux, bottom concentration-boil up) with weak nondynamic feed forward following a step change in feed flow $\left(v_{1}\right)$.

\section{Conclusion}

This investigation shows that the END principle yields superior control quality compared to conventional approaches both with regard to the suppression of 

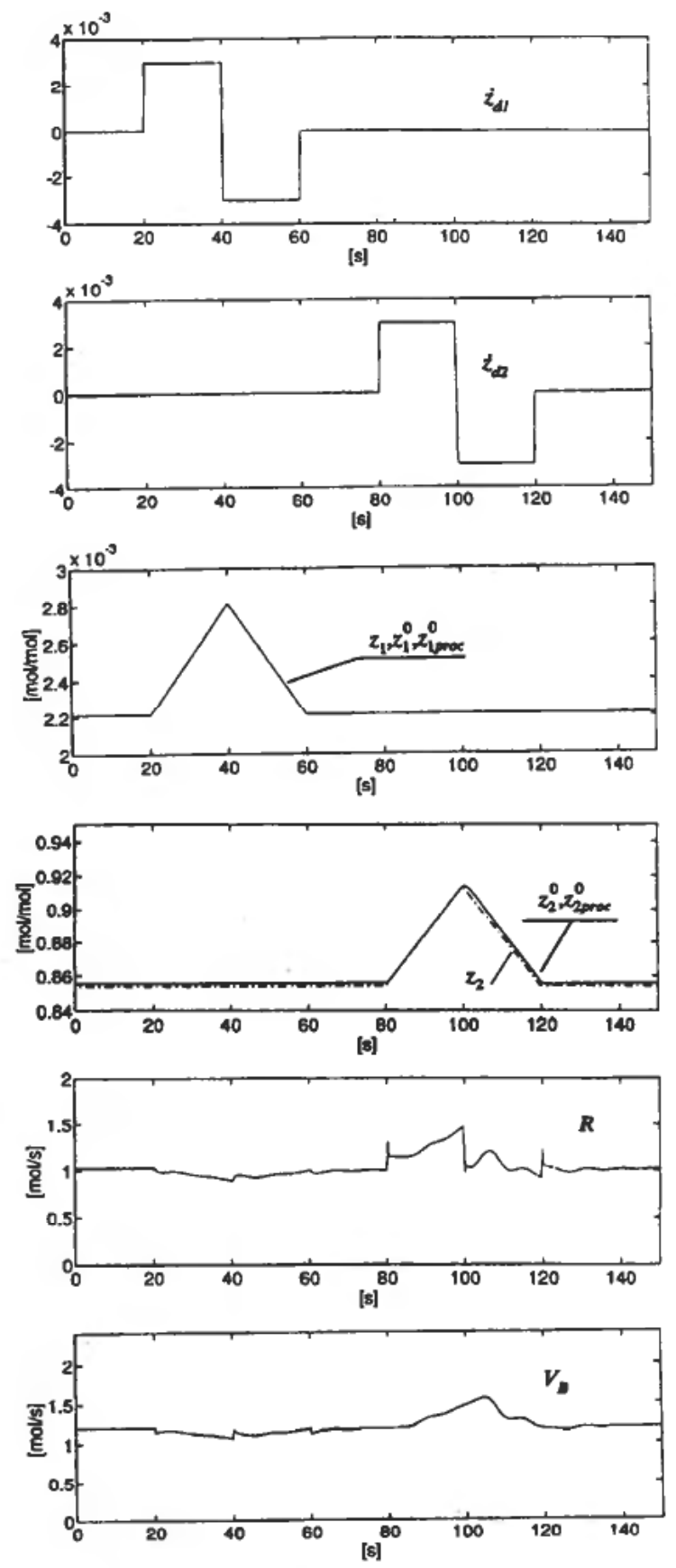

Figure 5(a). Nonlinear decoupling responses, step changes in $\dot{\boldsymbol{z}}_{d}$. 

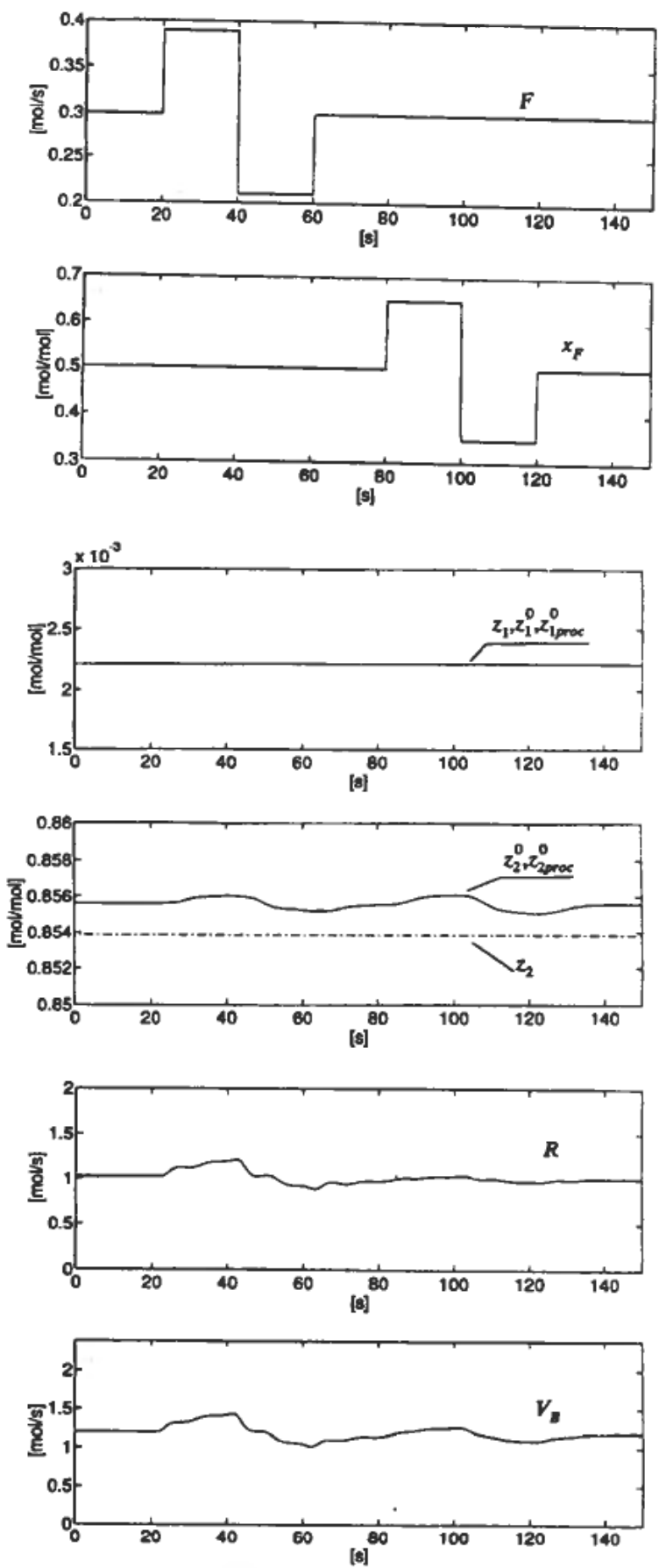

Figure 5(b). Nonlinear decoupling responses, step changes in $v$. 

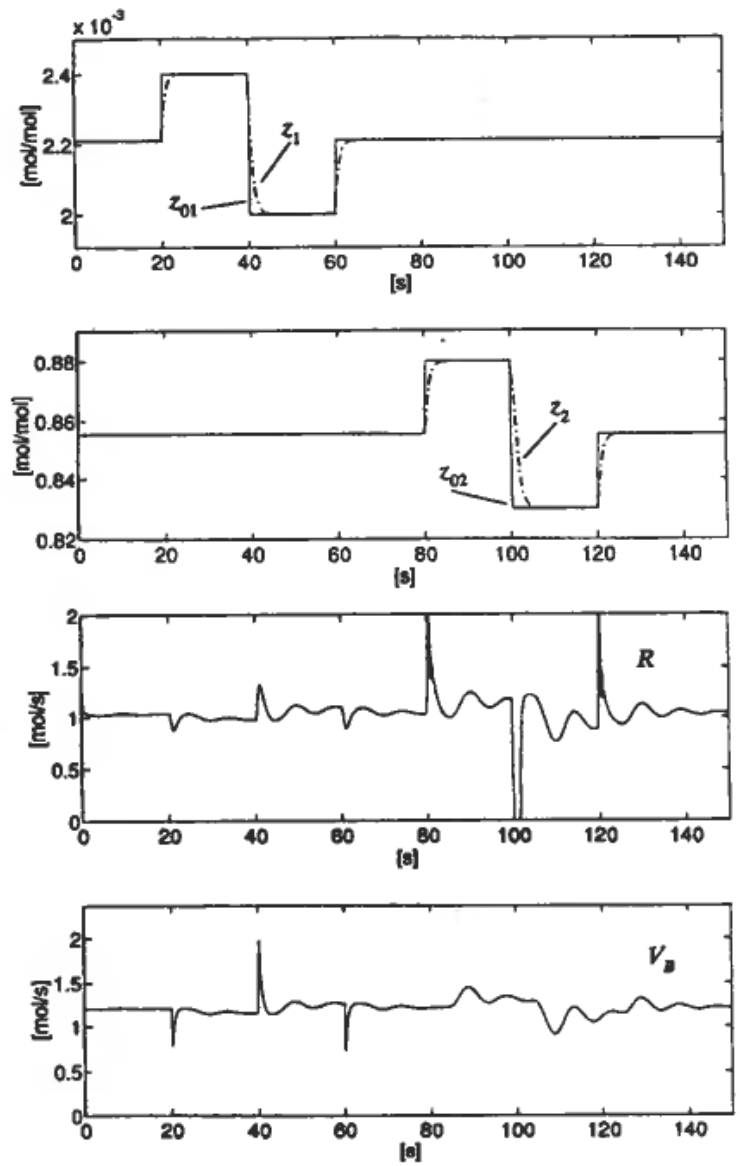

Figure 6. Loop I responses.

disturbances and following set point changes. Further studies of the influence of different kinds of model errors are suggested.

\section{ACKNOWLEDGMENT}

The simulation study reported is in part (BS) supported by STATOIL and (JGB) by SINTEF.

\section{Appendix}

The model to be used here is developed in Di Ruscio (1987) and uses standard self-explanatory notation as follows:

Reboiler stage

$$
\begin{gathered}
\dot{B}=K_{B}\left(L_{1}-V_{B}-B\right), \\
M_{B}=M_{0 B}+B / K_{B} \\
M_{B} \dot{x}_{B}=L_{1} x_{1}-V_{B} y_{B}-B x_{B}-\dot{M}_{B} x_{B}
\end{gathered}
$$

Tray 1

$$
\dot{L}_{1}=K_{1}\left(L_{2}-L_{1}+V_{B}-V_{2}\right)=K_{1}\left(L_{2}-L_{1}\right)
$$




$$
\begin{gathered}
M_{1}=M_{01}+L_{1} / K_{1} \\
M_{1} \dot{x}_{1}=L_{2} x_{2}-L_{1} x_{1}+V_{B} y_{B}-V_{1} y_{1}-\dot{M}_{1} x_{1}
\end{gathered}
$$

Tray $i=2, \ldots, f-1, f-2, \ldots, N-1$

$$
\begin{gathered}
\dot{L}_{i}=K_{i}\left(L_{i+1}-L_{i}+V_{i-1}-V_{i}\right) K_{i}\left(L_{i+1}-L\right) \\
M_{i}=M_{0 i}+L_{i} / K_{i} \\
M_{i} \dot{x}_{i}=L_{i+1} x_{i+1}-L_{i} x_{i}+V_{i-1} y_{i-1}-V_{i} y_{i}-\dot{M}_{i} x_{i}
\end{gathered}
$$

Feed tray $i=f$

$$
\begin{gathered}
\dot{L}_{i}=K_{f}\left(L_{i+1}-L_{f} V_{f-1}-V_{f}+F\right)=K_{f}\left(L_{f+1}-L_{f}+F\right) \\
M_{f}=M_{0 f}+L_{f} / K_{f} \\
M_{f} \dot{x}_{f}=L_{f+1} x_{f+1}-L_{f} x_{f}+V_{f-1} y_{f-1}-V_{f} y_{f}+F x_{F}-M_{f} x_{f}
\end{gathered}
$$

Top tray $i=N$

$$
\dot{L}_{N}=K_{N}\left(-L_{N}+V_{N-1}-V_{N}+R\right)
$$
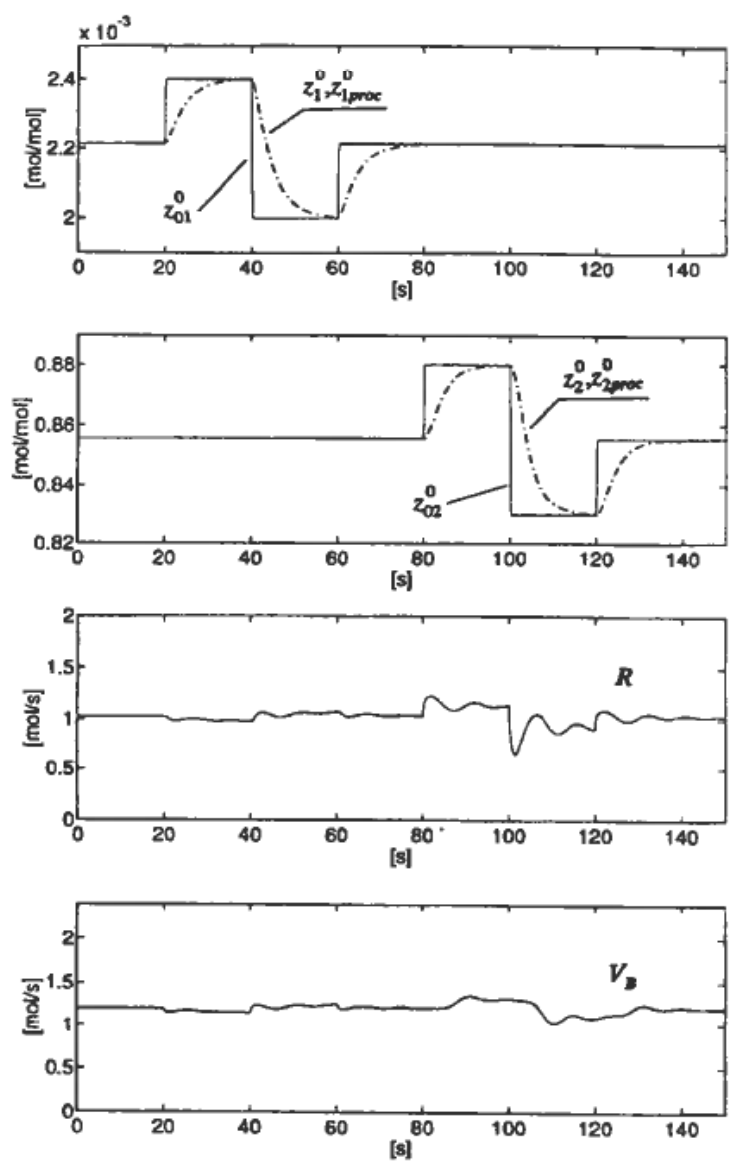

Figure 7(a). Loop II responses, stepchanges in $z_{0}^{0}$. 

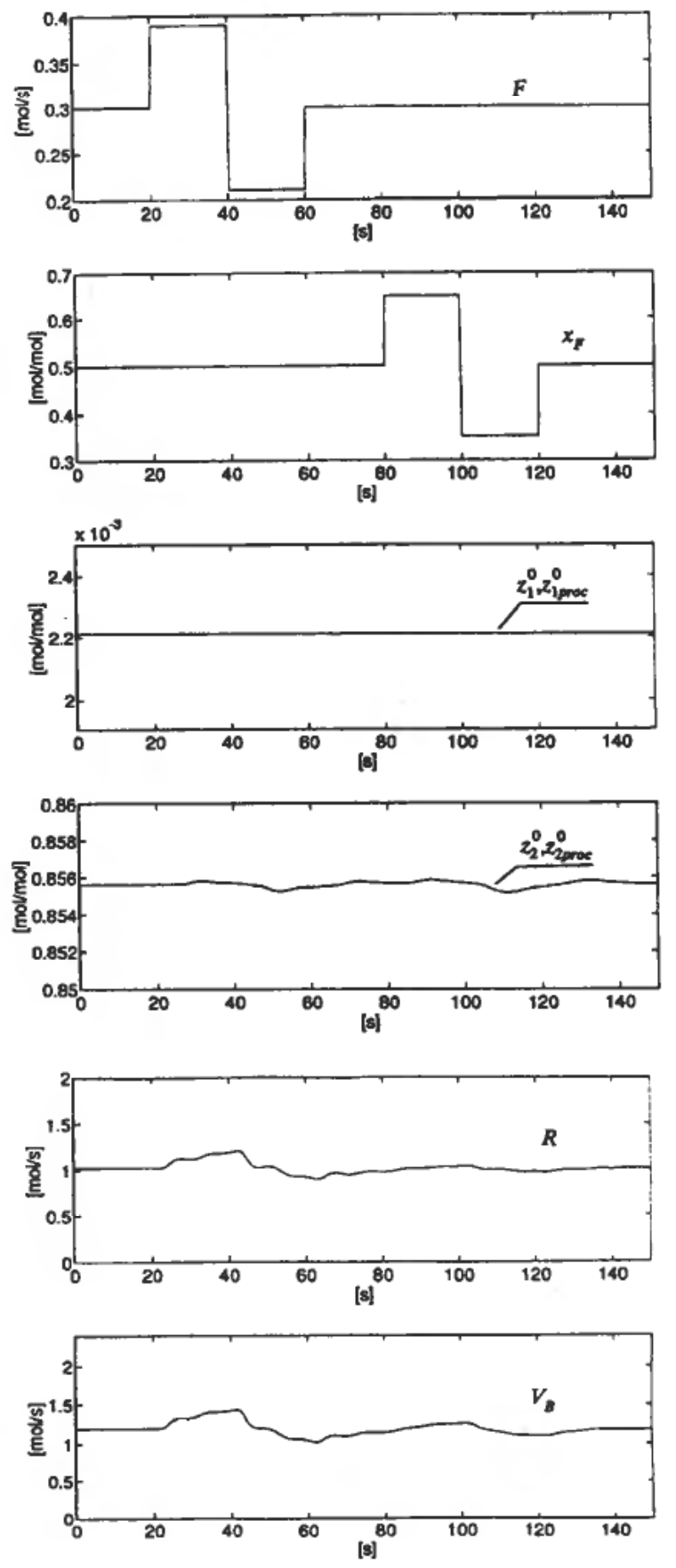

Figure $7(b)$. Loop II responses, step changes in $v$. 

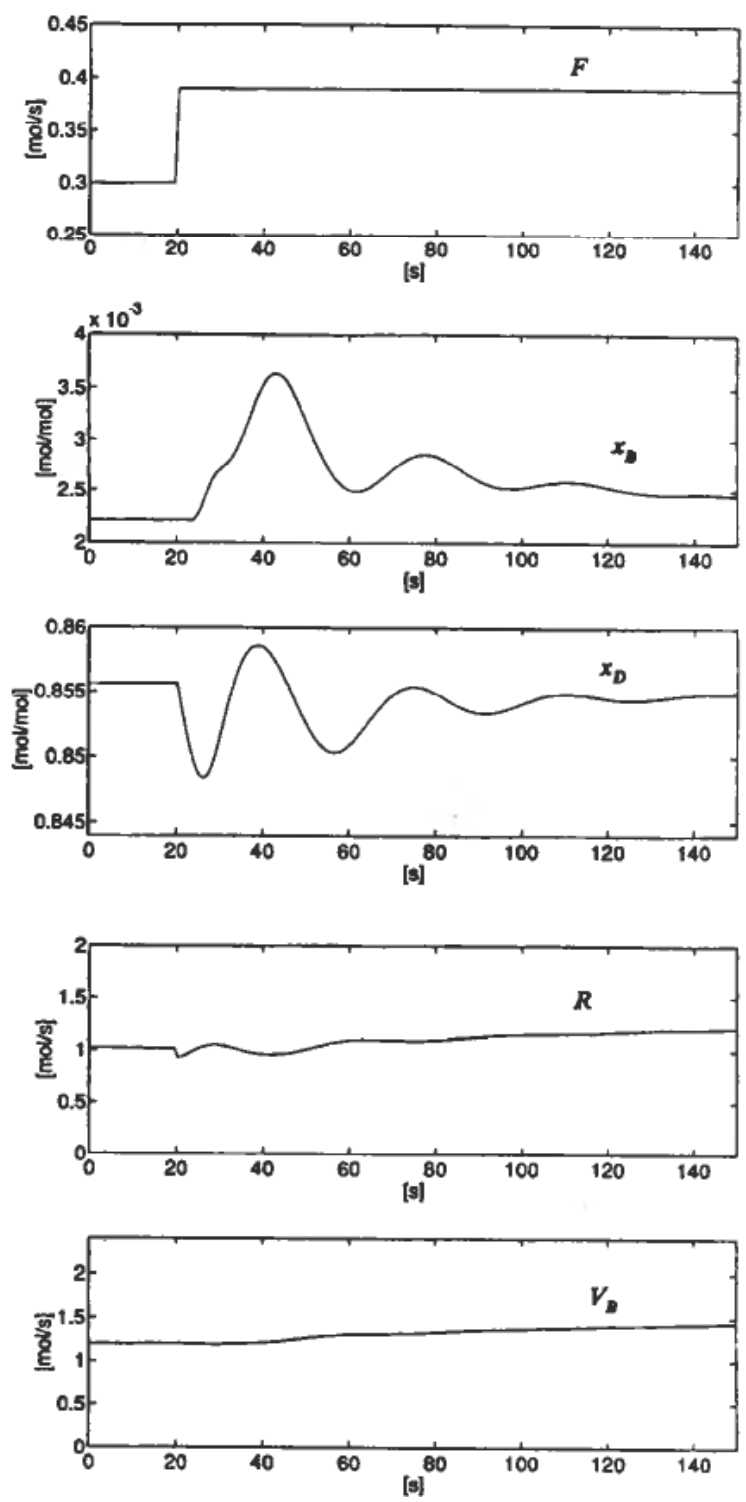

Figure 8. Conventional control system responses.

$$
\begin{gathered}
\dot{L}_{N}=K_{M}\left(-L_{N}+V_{N-1}-V_{N}+R\right) \\
M_{N}=M_{0 N}+L_{N} / K_{N} \\
M_{N} \dot{x}_{N}=-L_{N} x_{N}+V_{N-1} y_{N-1}-V_{N} y_{N}+R x_{D}-\dot{M}_{N} x_{N}
\end{gathered}
$$

Accumulator

$$
\begin{gathered}
\dot{D}=K_{D}\left(V_{N}-(R+D)\right) \\
M_{D}=M_{0 D}+D / K_{D} \\
M_{D} \dot{x}_{D}=V_{N} y_{N}-(R+D) x_{D}-\dot{M}_{D} x_{D}
\end{gathered}
$$


Equilibrium relationship between vapour and liquid concentration is assumed by

$$
y_{i}=\frac{\alpha x_{i}}{1+(\alpha-1) x_{i}}
$$

where $\alpha$ : relative volatility.

The model consists of $2 N+4$ differential equations and $2 N+3$ algebraic equations.

Parameters which have to be specified in the model are:

Number of trays, $N=10$

Column feed tray, $f=5$

Relative volatility, $\alpha=2.5$

Tray constant, $K_{i}=1(1 / \mathrm{s})$

Liquid mass in reboiler, $M_{0 B}=7.2(\mathrm{Mol})$

Liquid mass on tray, $M_{0 i}=1.8(\mathrm{Mol})$

Liquid mass in accumulator, $M_{0 D}=1 \cdot 1(\mathrm{Mol})$

The manipulated variables are:

Reflux, $R=1.025(\mathrm{Mol} / \mathrm{s})\left(u_{1}\right)$

Vapour flow from reboiler, $V_{\mathrm{B}}=1.2(\mathrm{Mol} / \mathrm{s})\left(u_{2}\right)$

Disturbances:

Feed flow, $F=0.3(\mathrm{Mol} / \mathrm{s})\left(v_{1}\right)$

Feed concentration, $x_{F}=0.5(\mathrm{Mol} / \mathrm{Mol})\left(v_{2}\right)$

$$
\begin{aligned}
& z_{1}=x_{B} \\
& z_{2}=x_{D}+d_{2 \cdot 23} D
\end{aligned}
$$

The $B(x)$ and $D$ matrices are as follows

$$
B(x)=\left[\begin{array}{cccccc}
0 & 0 & 0 \ldots 0 & b_{23 \cdot 1} & 0 \\
b_{1 \cdot 2} & b_{2 \cdot 2} & 0 \ldots & 0 & 0 & 0
\end{array}\right]^{T}
$$

where the nominal values are:

$$
\begin{aligned}
& b_{22 \cdot 1}=0.05, b_{23 \cdot 1}=-1 \cdot 0, b_{1 \cdot 2}=-1, b_{2 \cdot 2}=-0.045
\end{aligned}
$$

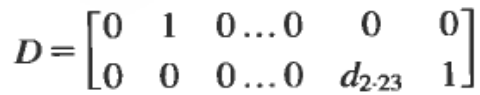

where

$$
\begin{aligned}
& d_{2 \cdot 23}=-0.01: \text { implemented, } \\
& d_{2 \cdot 23}=+0 \cdot 01: \text { tested in Fig. } 3 .
\end{aligned}
$$

\section{REFERENCES}

BALCHEN, J. G. (1991). Nonlinear decoupling in process control, Modeling, Identification and Control, 12, 81-94.

BALCHEN, J.G. (1993). Design of the property transformation in elementary nonlinear decoupling of multivariable processes. Modeling, Identification and Control, 14, 219-227.

BALCHEN, J. G., LIE, B. and SOLBERG, I. (1987). Internal decoupling in non-linear process control, 10th IFAC World Congress on Automatic Control, Munich, 1987; also Modeling, Identification and Control, 1988, 9, 137148. 
Di Ruscio, D. (1987). Dynamic mathematical models for a binary distillation process. (In Norwegian.) Report 87-64-U. Dept. of Engineering Cybernetics, The Norwegian Institute of Technology, Trondheim.

IsIDORI, A. (1989). Nonlinear Control System (Springer-Verlag, Berlin).

SKogestad, S. (1992). Dynamics and control of distillation columns-A critical review. Proc. IFAC symp. DYCORD'92 (J. G. Balchen, Ed.), IFAC Symposia Series no. 2 (Pergamon Press, London). 